\title{
Application of random walk model to fit temperature in 46 gamma world cities from 1901 to 1998
}

\author{
Shaomin $\operatorname{Yan}^{1}$, Guang $\mathrm{Wu}^{1,2^{\star}}$ \\ ${ }^{1}$ State Key Laboratory of Non-food Biomass Enzyme Technology, National Engineering Research Center for Non-food Biorefinery, \\ Guangxi Key Laboratory of Biorefinery, Guangxi Academy of Sciences, Nanning, China \\ ${ }^{2}$ DreamSciTech Consulting, Shenzhen, China; ${ }^{*}$ Corresponding Author: hongguanglishibahao@yahoo.com
}

Received 2 October 2010; revised 3 November 2010; accepted 6 November 2010.

\begin{abstract}
Very recently, we have applied the random walk model to fit the global temperature anomaly, CRUTEM3. With encouraging results, we apply the random walk model to fit the temperature walk that is the conversion of recorded temperature and real recorded temperature in 46 gamma world cities from 1901 to 1998 in this study. The results show that the random walk model can fit both temperature walk and real recorded temperature although the fitted results from other climate models are unavailable for comparison in these 46 cities. Therefore, the random walk model can fit not only the global temperature anomaly, but also the real recorded temperatures in various cities around the world.
\end{abstract}

Keywords: Gamma World Cities; Global Warming; Modeling; Random Walk; Temperature Change

\section{INTRODUCTION}

During our recent studies on the potential influence of global warming on the evolution of proteins from influenza A virus [1-5], we noticed that the temperature fluctuates around the temperature trend. On the one hand, these fluctuations can be easily attributed to random effects; on the other hand, these fluctuations might imply that we might need a random model to describe the temperature pattern because the output of any deterministic model is generally a smooth curve.

Without smooth temperature trend, the temperature behaves irregularly either to increase or to decrease for some period of time, from where we could not find out a clear pattern in temperature change along the time course. The irregular ups and downs appear similar to the random walk, which firstly came into our mind. The random walk comes from the observation of tossing a single coin: although theoretically each side of coin has 0.5 chances to be up or down, the tossing of coin sequentially results in either head up or tail up for several times continuously rather than each tossing generates alternative result. The addition of sequentially tossed results would be a random walk [6]. Computationally each tossing of coin can be done with the generation of random numbers, which can be classified as 1 or -1 if a random number is larger or smaller than its previous one. As the generation of random numbers is through the Monte-Carlo simulation with different seeds, thus the random walk is a model with its own model parameter, seed.

Therefore, we can consider the random walk model as alternative model to fit the temperature because it can produce a really fluctuated curve. Following that, we applied the random walk model to fit the global temperature anomaly, CRUTEM3, and got a very encouraging result [7]. Since then, an intensive literature search indicates that Gordon noticed the similarity between global warming and random walk in 1991 [8].

Likely, the random walk could provide an alternative model to describe the temperature, however, two questions raised here are whether the random walk model can be applied only to the global temperature anomaly not to local temperature and whether the random walk model can be applied only to the anomaly not to really recorded temperature.

In order to answer these questions, we need to use the random walk model to fit the real recorded temperature in different places around the world. In this study, we apply the random walk model to fit the temperature in 46 gamma world cities from 1901 to 1998.

\section{MATERIALS AND METHODS}

\subsection{Data}

Forty-six gamma world cities are chosen according to 
Wikipedia [9]. However, the number of these cities and their order are changed frequently due to the characteristics of Wikipedia, thus the cities were dated in February, 2010.

The temperatures recorded in these 46 cities from 1901 to 1998 based on $0.5^{\circ}$ by $0.5^{\circ}$ latitude and longitude grid-box basis cross globe are obtained from the website of Oak Ridge National Laboratory [10].

The latitudes and longitudes of these 46 gamma world cities are determined using Get Lat Lon [11].

\subsection{Temperature Walk}

At first, we use the simplest random walk model, which starts at zero and at each step moves by \pm 1 with equal probability [6]. In other words, the simplest random walk can be considered as a sequential result of tossing a fair coin, by which we record the head as 1 and the tail as -1 , and then we add the results along the time course.

For this purpose, we need to convert the temperature into the temperature walk as shown in Table 1. When the temperature at certain year is higher than its previous one, we classify it as 1 , otherwise we classify it as -1 , and then we add them as the random walk does.

\subsection{Generation of Random Walk}

We use the SigmaPlot [12] to generate random sequence for the random walk. Technically, the generation of random walk is quite simple: we generate random number either ranged from -1 to 1 or without limit, and then we classify a generated random number as 1 if it is larger than its previous one and as -1 if it is smaller than its previous one. Thereafter we add these values as a random walk.

\subsection{Searching for Seed}

To find a random walk that is very approximate to the temperature walk is to find a seed that can generate such a random walk. To the best of our knowledge, there is no algorithm for searching seeds by converging the difference between observed curve and the curve produced by random walk. Therefore the so-called fitting, which traditionally searches the optimum according to various algorithms, becomes to search all possible seeds in order to find out the seed that produces the random walk with the least squares between random walk and temperature walk.

\subsection{Fitting Recorded Temperature}

Thereafter, we use a more complicated random walk model [13] to fit the recorded temperature, which is in decimal format. In plain words, the simplest random walk comes from tossing of double-sided coin, while this random walk can be regarded as tossing of dice, which cannot be only six-sided but as many as the decimal data. In such a way, we generate random numbers, and add them to construct the random temperature, and the fitting is again to search the best seed that generates the best fit.

\subsection{Comparison}

We use the least squares between temperature walk and random walk, and between recorded temperature and random temperature to evaluate which seed is the best.

\section{RESULTS AND DISCUSSION}

Table 1 shows how we construct a temperature walk in Panama City. Its recorded temperature in 1901 was 18.8250 (cell 2, column 2), which corresponds to the starting point of temperature walk, 0 , (cell 2, column 4). The temperature in 1902 was 19.8250 (cell 3, column 2), which was higher than the temperature in 1901, 18.8250, thus the temperature step was 1 (cell 3 , column 3 ), and the temperature walk is $1(0+1)$ (cell 3 , column 4$)$. In this manner, we construct the temperature walk from 1901 to 1998.

Similarly, Table $\mathbf{1}$ also shows how we construct a random walk with generated random numbers. A good seed that we found is 0.48531 . The first random number generated by this seed was 0.2629 (cell 2, column 5), which corresponds to the starting point of random walk, 0 , (cell 2, column 7). The second random number generated was 0.8817 (cell 3, column 5), which is larger than the first random number, 0.2629 (cell 2, column 5). Thus the random step was 1 (cell 3, column 6), and the random walk is $1(0+1)$ (cell 3 , column 7$)$.

The last column (column 8) in Table $\mathbf{1}$ is the difference between temperature walk and random walk (random walk-temperature walk), whose squared sum is our standard to find the best fit among seeds.

Figure 1 shows the fitted results in 12 cities represented differently geographic locations around the world. As can be seen, the random walk (gray curve) mimicked the temperature walk (black curve) with very small difference. Theoretically, a completely perfect fit would have an extremely small probability. In the simplest case of random walk, this probability would be $(1 / 2)^{98}$. Meanwhile, the total number of our fittings were one million, which is a fraction of $(1 / 2)^{98}$. Thus the fact that we can find a relatively good fit within one million fittings suggests that the random walk can describe the temperature pattern from 1901 to 1998 in these cities. 


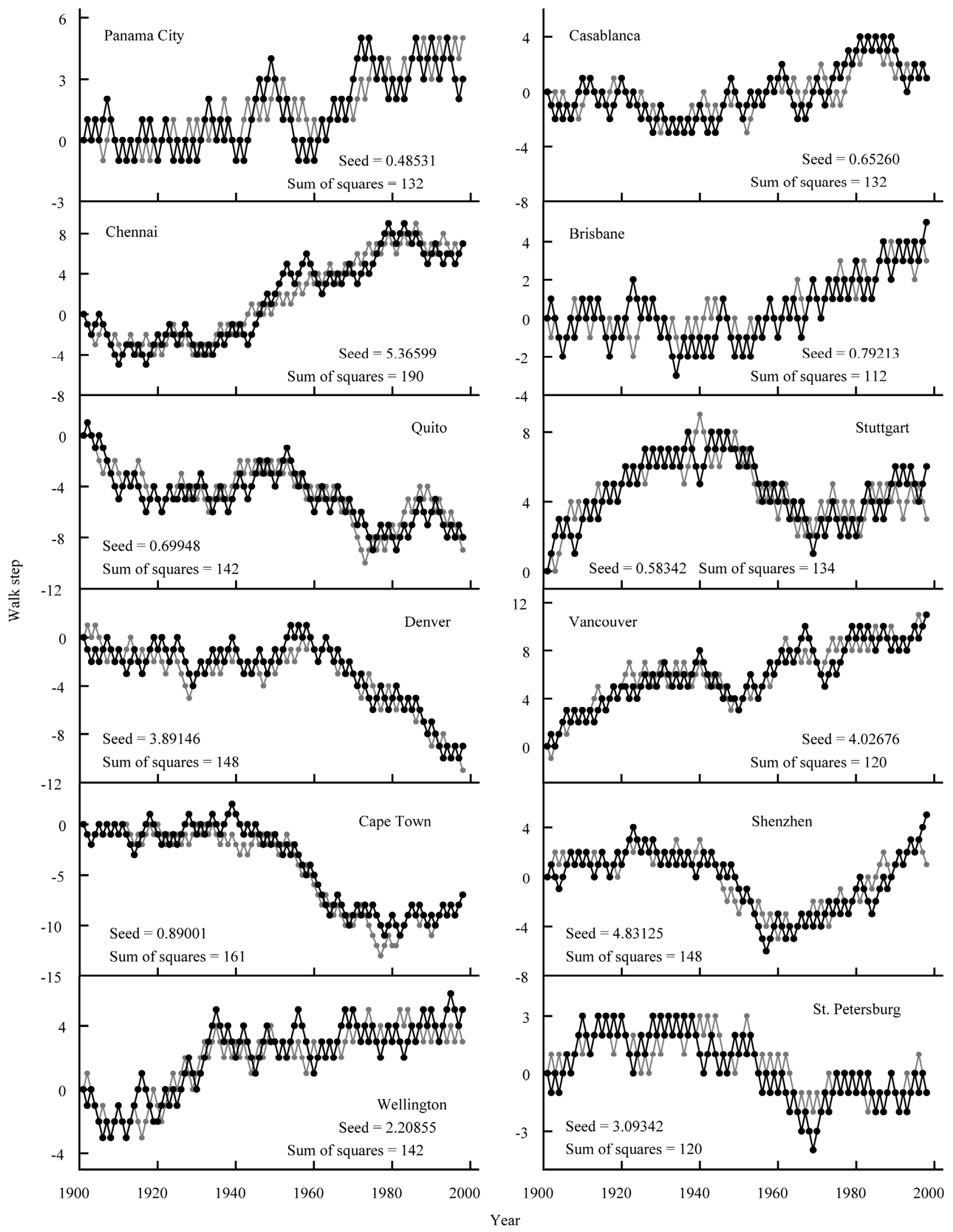

Figure 1. Comparison of temperature walk (black) with random walk (gray) in 12 cities from 1901 to 1998. 
Table 1. Conversion of recorded temperature in centigrade degree into temperature walk and generation of random walk for temperature in Panama City from 1901 to 1998 (The random number is generated by SigmaPlot with the seed of 0.48531).

\begin{tabular}{|c|c|c|c|c|c|c|c|}
\hline Year & $\begin{array}{c}\text { Recorded } \\
\text { Temperature }\end{array}$ & Temperature Step & Temperature Walk & Random Number & Random Step & Random Walk & Difference \\
\hline 1901 & 18.8250 & & 0 & 0.2629 & & 0 & 0 \\
\hline 1902 & 19.8250 & 1 & 1 & 0.8817 & 1 & 1 & 0 \\
\hline 1903 & 19.2250 & -1 & 0 & 0.2996 & -1 & 0 & 0 \\
\hline 1904 & 19.8000 & 1 & 1 & 0.9392 & 1 & 1 & 0 \\
\hline 1905 & 19.7750 & -1 & 0 & -0.1281 & -1 & 0 & 0 \\
\hline$\cdots$ & $\cdots$ & $\cdots$ & $\ldots$ & $\ldots$ & $\ldots$ & $\cdots$ & \\
\hline 1991 & 20.7083 & -1 & 4 & 0.4440 & 1 & 4 & 0 \\
\hline 1992 & 20.0333 & -1 & 3 & 0.5661 & 1 & 5 & 2 \\
\hline 1993 & 20.0750 & 1 & 4 & -0.8129 & -1 & 4 & 0 \\
\hline 1994 & 20.4750 & 1 & 5 & 0.0808 & 1 & 5 & 0 \\
\hline 1995 & 20.2250 & -1 & 4 & -0.8109 & -1 & 4 & 0 \\
\hline 1996 & 19.6917 & -1 & 3 & -0.1161 & 1 & 5 & 2 \\
\hline 1997 & 19.6750 & -1 & 2 & -0.9795 & -1 & 4 & 2 \\
\hline 1998 & 20.7333 & 1 & 3 & -0.3824 & 1 & 5 & 2 \\
\hline
\end{tabular}

On the other hand, the temperature walk in fact answers the simplest and very basic question of whether the temperature in this year is higher (1) or lower $(-1)$ than that in the previous year, which could arguably be the first human concept in comparison of temperature between two time points. The answer to this simplest question for years would construct the temperature walk. Therefore, we consider that the temperature walk has a very sound basis.

In fact, not only the selected cities in Figure 1 show a good fit, but also the rest of cities were found no exception (Table 2), here the exception can be understood as the fitted curve has a totally opposite direction with respect to its temperature curve. We therefore consider that the random walk model can fit not only the global temperature anomaly, but also the temperatures in different locations around the world.

Figure 2 shows the fittings for the recorded temperature. The difference between Figures $\mathbf{1}$ and $\mathbf{2}$ is that the recorded temperature was in decimal format while the temperature walk was in \pm 1 format. As seen in Figure 2, the random walk model can fit these recorded temperatures even the temperatures in these locations have different patterns. Again, there is no exception for the fittings in all 46 gamma world cities (columns 3 and 5, Table 2), which furthermore demonstrate that the random walk model can fit not only the global temperature anomaly, but also the temperatures in different locations around the world.

In this study, we used the sum of least squares to com- pare which seed produces a better fitting. Thus, a question raised here is how small the sum of least squares is that we can say the random walk model fits the temperature well. We could consider this question in three ways: 1) We have already mentioned that the probability to find the best fit for the temperature walk is $(1 / 2)^{98}$, and this chance cannot be found in limited time, however, some better fits can be found among our total one million fits; 2) On the other hand, the sum of least squares can be mainly used for model comparison, i.e. to use our sum of least squares to compare with the sum of least squares obtained from other models in fitting the temperature of this group of cities although no other results are available for comparison; 3) from modeling viewpoint, the random walk model has an advantage over other models because the random walk model has only one model parameter, whereas other models have numerous model parameters, thus theoretically the random walk model has a larger chance than other models to find out the best fit because each model parameter has a certain space to search the optimal parameter.

The random walk actually has a very deeply physical mechanism: weather is a highly multidimensional attractor of Navier-Stokes Equations of atmosphere with highly complicated boundary conditions. Worse, it is a non-autonomois system with at least two-periodic forcing, diurnal and annual. And annual measurement is a result of averaging of diurnal Poincare Maps. Given what we know about weather, this entire attractor is highly chaotic, its annual maps are likely of very fractal 


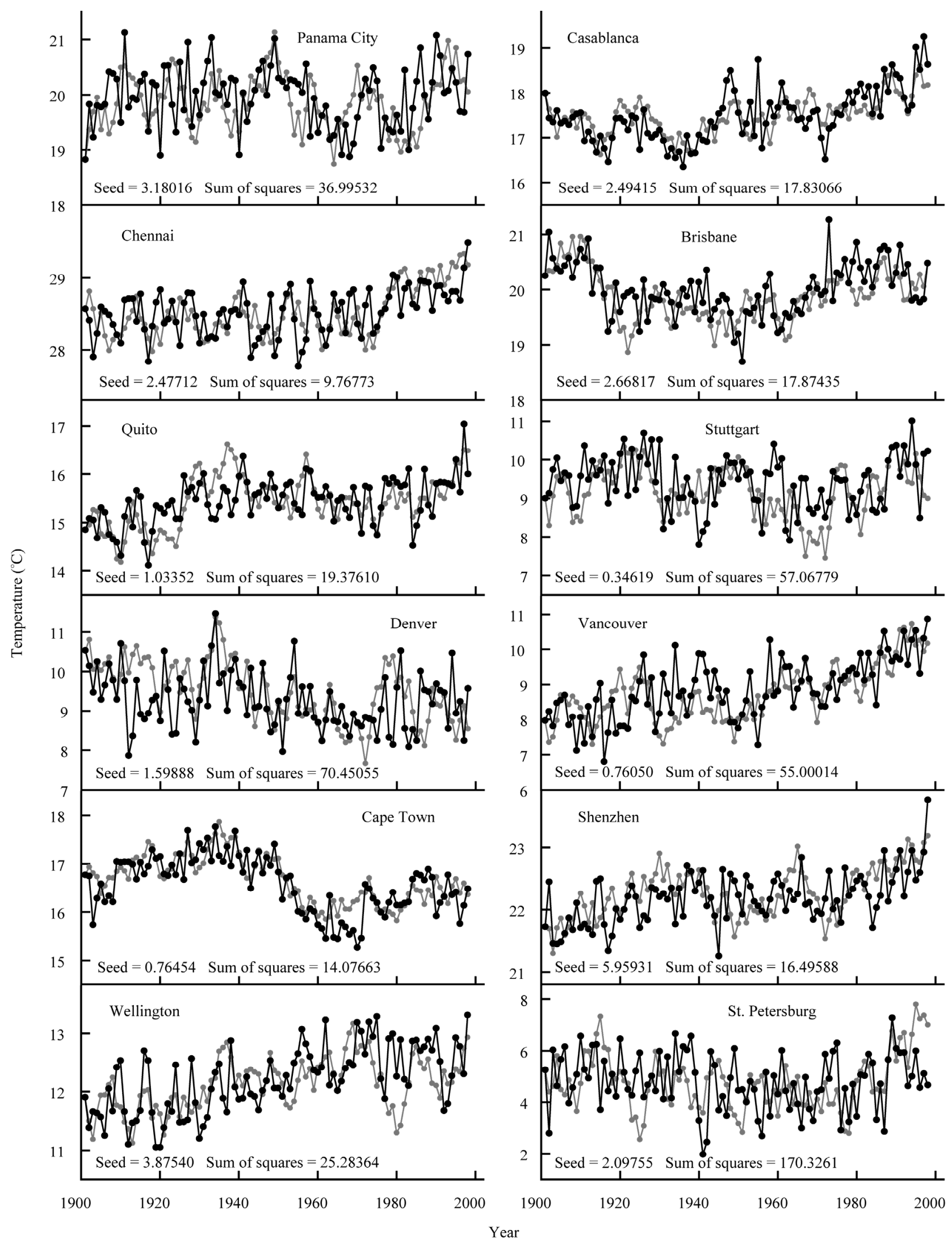

Figure 2. Comparison of recorded temperature (black) with random temperature (grey) in 12 cities from 1901 to 1998. 
Table 2. Model parameters (seeds) and fitted results for fitting temperature change in 46 gamma world cities from 1901 to 1998 using random walk model.

\begin{tabular}{|c|c|c|c|c|}
\hline \multirow{2}{*}{ City } & \multicolumn{2}{|c|}{ Fitting of Temperature Walk } & \multicolumn{2}{|c|}{ Fitting of Recorded Temperature } \\
\hline & Seed & Sum of Squares & Seed & Sum of Squares \\
\hline Panama City & 0.48531 & 132 & 3.18016 & 36.99532 \\
\hline Casablanca & 0.65260 & 132 & 2.49415 & 17.83066 \\
\hline Chennai & 5.36599 & 190 & 2.47712 & 9.76773 \\
\hline Brisbane & 0.79213 & 112 & 2.66817 & 17.87435 \\
\hline Quito & 0.69948 & 142 & 1.03352 & 19.37610 \\
\hline Stuttgart & 0.58342 & 134 & 0.34619 & 57.06779 \\
\hline Denver & 3.89146 & 148 & 1.59888 & 70.45055 \\
\hline Vancouver & 4.02676 & 120 & 0.76050 & 55.00014 \\
\hline Zagreb & 1.90779 & 116 & 8.66967 & 51.31698 \\
\hline Guatemala City & 0.24543 & 152 & 1.83630 & 16.95153 \\
\hline Cape Town & 0.89001 & 161 & 0.76454 & 14.07663 \\
\hline San Jose & 1.02726 & 97 & 0.14315 & 27.59630 \\
\hline Ljubljana & 1.9037 & 148 & 3.84243 & 42.53592 \\
\hline Minneapolis & 0.35895 & 152 & 0.36478 & 109.71508 \\
\hline Santo Domingo & 4.29052 & 132 & 1.74614 & 12.86316 \\
\hline Seattle & 1.42289 & 116 & 0.25169 & 39.26277 \\
\hline Manama & 2.02165 & 124 & 0.68838 & 27.38144 \\
\hline Shenzhen & 4.83125 & 148 & 5.95931 & 16.49588 \\
\hline Guadalajara & 2.04735 & 146 & 0.36390 & 23.99288 \\
\hline Antwerp & 6.65174 & 140 & 2.48303 & 46.55537 \\
\hline Kolkata & 9.56581 & 134 & 5.27468 & 11.90250 \\
\hline Rotterdam & 0.11644 & 132 & 2.48303 & 44.69400 \\
\hline Lagos & 1.77962 & 144 & 1.69805 & 23.25883 \\
\hline Philadelphia & 1.16765 & 120 & 3.33953 & 53.14891 \\
\hline Perth & 0.03095 & 131 & 0.75289 & 26.84818 \\
\hline Amman & 1.41563 & 136 & 0.58696 & 30.47234 \\
\hline Manchester & 2.64776 & 113 & 2.66954 & 32.74658 \\
\hline Riga & 3.64228 & 112 & 2.83735 & 144.92674 \\
\hline Detroit & 0.00136 & 124 & 0.01219 & 85.23191 \\
\hline Guayaquil & 0.72301 & 139 & 3.97256 & 25.55697 \\
\hline Wellington & 2.20855 & 142 & 3.87540 & 25.28364 \\
\hline Portland & 0.28335 & 132 & 0.07853 & 49.35406 \\
\hline Porto & 1.92792 & 133 & 0.38211 & 20.36737 \\
\hline Edinburgh & 0.77095 & 104 & 0.97533 & 28.76731 \\
\hline Tallinn & 0.16784 & 132 & 2.83735 & 147.22377 \\
\hline San Salvador & 1.06139 & 184 & 3.05414 & 16.39708 \\
\hline St. Petersburg & 3.09342 & 120 & 2.09755 & 170.32610 \\
\hline Port Louis & 0.08480 & 136 & 3.02959 & 6.53177 \\
\hline San Diego & 2.18277 & 250 & 0.12773 & 44.65777 \\
\hline Calgary & 1.37753 & 100 & 1.11564 & 139.44530 \\
\hline Almaty & 2.39794 & 164 & 9.93285 & 93.91648 \\
\hline Birmingham & 0.45373 & 142 & 0.95604 & 53.31933 \\
\hline Islamabad & 5.62339 & 110 & 0.91978 & 33.98368 \\
\hline Doha & 0.64310 & 135 & 0.70169 & 27.9417 \\
\hline Vilnius & 0.33912 & 116 & 0.53868 & 130.0170 \\
\hline Colombo & 7.06029 & 184 & 2.74576 & 5.83075 \\
\hline
\end{tabular}


nature, and invariant measure of these maps are likely a fractal-dimensional sieves with a hyperbolic point at every location. The annual iteration of this map is like a coin tossing [14].

Still, the random walk could be viewed as the Drunkard's walk [15] as the climate change seems to be a complex process, and the drunkard walk is also used to describe complex processes. It should be obvious that climate change is a complex process, for the climate change involves many factors that are not reducible to exactly one thing. And the climate affects the ecosystem that is a complex process itself.

On the other hand, the random mechanism in random walk model is different from other random factors in modeling, where the random factors were mainly considered as a minor factor [16].

At this moment, we have no way to know whether or not the random walk model works better than other climate models, simply because the results of temperature fitting in these cities using other climate models are not available for comparison.

In conclusion: we use the random walk model to fit the temperature change in 46 gamma world cities from 1901 to 1998 , and the results show that the random walk model can fit both the temperature walk and recorded temperature in different cities worldwide. This study confirms that the random walk model can be used to analyze the temperature change, which suggests that the random mechanism could be a factor driving the temperature change in these cities. The use of random walk to fit the temperature change is still at very early stage nevertheless more studies are needed in order to better understand the temperature change and its modeling.

\section{ACKNOWLEDGEMENTS}

This study was partly supported by Guangxi Science Foundation (07-109-001A, 08-115-011, 09322001 and 2010GXNSFA013046). The authors wish to thank Dr Hong Zhang at Biyee SciTech Inc., MA, USA for helpful discussion. The authors also wish to thank Dr Alexei A. Predtechenski at Standard Microsystems Corporation, Austin, TX, USA for his discussion and support in internet discussion group.

\section{REFERENCES}

[1] Yan, S. and Wu, G. (2009) What these trends suggest?
American Journal of Applied Sciences, 6, 1116-1121.

[2] Yan, S. and Wu, G. (2009) Trends in global warming and evolution of polymerase basic protein 2 family from influenza A virus. Journal of Biomedical Sciences and Engineering, 2, 458-464.

[3] Yan, S. and Wu, G. (2009) Trends in global warming and evolution of matrix protein 2 family from influenza A virus. Interdisciplinary Sciences: Computational Life Sciences, 1, 272-279.

[4] Yan, S.M., Zuo, W.P., Zhu, Q.X., Huang, Y.Y., Pan, L.X. and Wu, G. (2010) Trends in global warming and evolution of neuraminidases from influenza A viruses since 1918. Guangxi Sciences, 17, 80-84.

[5] Yan, S. and Wu, G. (2010) Trends in global warming and evolution of nucleoproteins from influenza A viruses since 1918. Transboundary and Emerging Diseases.

[6] Feller, W. (1968) An introduction to probability theory and its applications. 3rd Edition, Wiley, New York.

[7] Yan, S. and Wu, G. (2010) Modeling of global temperature change from 1850 to 2009 using random walk. Guangxi Sciences, 17, 148-150.

[8] Gordon, A.H. (1991) Global warming as a manifestation of a random walk. Journal of Climate, 4, 589-597.

[9] Wikimedia Foundation, Inc. (2010) Global city. http://en.wikipedia.org/wiki/Global_city

[10] New, M., Hulme, M. and Jones, P. (2000) Representing twentieth-century space-time climate variability. Part II: Development of 1901-96 monthly grids of terrestrial surface climate. Journal of Climate, 13, 2217-2238.

[11] Willison, S. (2010) Get lat lon. http://www.getlatlon.com/

[12] SPSS Inc., SigmaPlot for Windows Version 8.02. (19862001).

[13] Borovkov, A. and Borovkov, K. (2008) Asymptotic analysis of random walks: heavy-tailed distributions, Cambridge University Press, Cambridge.

[14] Tekhasski, A. (2010) Random walk model suggests that the global warming could be mainly attributed to random mechanism. http://www.amazon.com/tag/science/forum/ ref $=$ cm_cd_pg_pg7?_encoding $=U T F 8 \& c d F o r u m=F x Z 58$ KVEERYS5E\&cdPage $=7 \& c d$ Sort $=$ oldest $\&$ cdThread $=$ Tx 3TXP04WUSD4R1\&displayType $=$ tagsDetail

[15] Neil, J. (2009) Simply complexity: A clear guide to complexity theory. Reprint edition, Oneworld Publications, Oxford.

[16] Williams, P.D. (2005) Modelling climate change: the role of unresolved processes. Philosophical Transactions of the Royal Society A, 363, 2931-2946. 